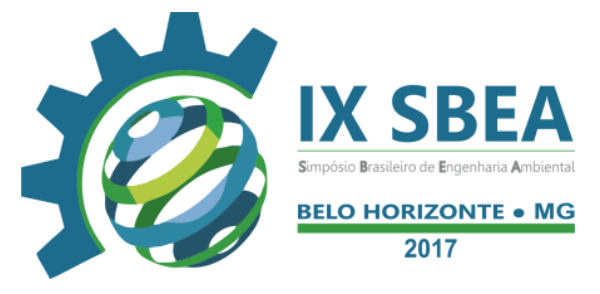

ÁREA TEMÁTICA: RESÍDUOS SÓLIDOS

\title{
A alta frequência de coleta de dados de temperatura como indicador de qualidade da compostagem de lodo de laticínio na presença e ausência de cinza de caldeira
}

\begin{abstract}
Andressa Ferreira Pimenta -drefpimenta@gmail.com
Universidade Tecnológica Federal do Paraná - UTFPR - Campus Londrina. Avenida dos Pioneiros, 3131 CEP 86036-370

Giovanni Terra Peixoto - gtpeixoto@gmail.com
\end{abstract}

Universidade Tecnológica Federal do Paraná - UTFPR - Campus Londrina. Avenida dos Pioneiros, 3131 CEP 86036-370

Marcos Candido da Silva - marcos.1993@alunos.utfpr.edu.br

Universidade Tecnológica Federal do Paraná - UTFPR - Campus Londrina. Avenida dos Pioneiros, 3131 CEP 86036-370

Tatiane Cristina Dal Bosco - tatianebosco@utfpr.edu.br

Universidade Tecnológica Federal do Paraná - UTFPR - Campus Londrina. Avenida dos Pioneiros, 3131 CEP 86036-370

Janksyn Bertozzi - janksynbertozzi@utfpr.edu.br

Universidade Tecnológica Federal do Paraná - UTFPR - Campus Londrina. Avenida dos Pioneiros, 3131 CEP 86036-370

Roger Nabeyama Michels - rogernmichels@utfpr.edu.br

Universidade Tecnológica Federal do Paraná - UTFPR - Campus Londrina. Avenida dos Pioneiros, 3131 CEP 86036-370

Elizabeth Mie Hashimoto - ehashimoto@utfpr.edu.br

Universidade Tecnológica Federal do Paraná - UTFPR - Campus Londrina. Avenida dos

Pioneiros, 3131 CEP 86036-370 

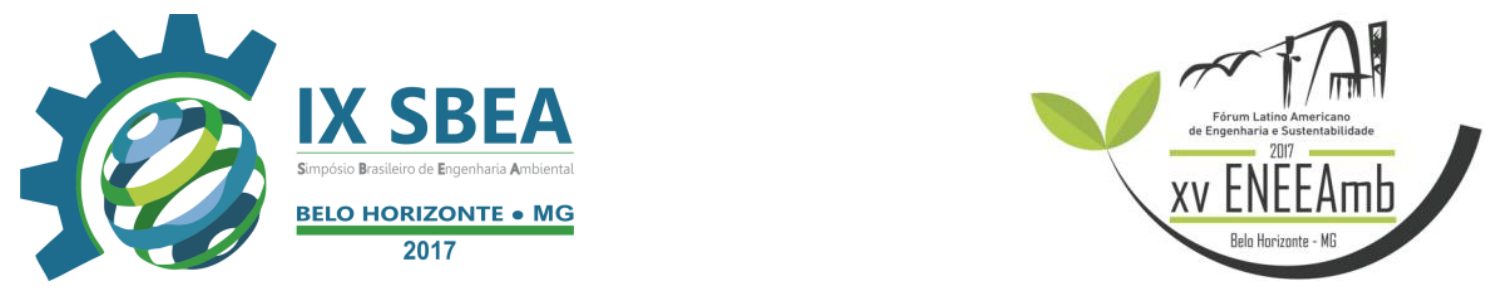

\section{RESUMO}

O tratamento biológico realizado na estação de tratamento de efluentes na indústria de laticínios gera ao final do processo o lodo biológico, que apresenta alto teor de matéria orgânica que se disposto incorretamente, pode causar degradação do meio ambiente. Deste modo, a compostagem é uma técnica utilizada no tratamento de resíduos sólidos orgânicos, que tem o intuito de diminuir significativamente o volume de resíduos durante o processo, tornando-os em um composto rico, e mais assimilável as plantas. O objetivo do trabalho é avaliar as temperaturas decorrentes dos dez primeiros dias de compostagem, sendo analisado a influência de cinza de caldeira na composição residual de leiras. Para realização no monitoramento das temperaturas duas leiras foram montadas, sendo elas: Leira 1, composta por lodo de laticínios (30L), capim (75L) e borra de café (45L); e Leira 2, contendo a mesma composição da Leira 1, porém com adição de cinza de caldeira (15L). As leiras foram montadas ao abrigo de chuva sobre piso impermeável e um sistema de coleta de temperatura automatizado foi instalado, em que cada leira transmitia, via sensor e a cada $15 \mathrm{~min}$., as temperaturas coletadas em três pontos distintos. Ao décimo dia de compostagem, realizou-se a leitura de $\mathrm{pH}$ do composto. Ambas as leiras atingiram fase termofílica após o quarto dia de compostagem. A leira com inserção de cinzas atingiu níveis mais altos de temperatura, chegando ao máximo de $54^{\circ} \mathrm{C}$ no sétimo dia, contra os $47^{\circ} \mathrm{C}$ atingidos pela leira sem adição de cinzas. Os valores de $\mathrm{pH}$ encontrados foram de 7,79 e 8,87, para as Leiras 1 e 2, respectivamente, o que está dentro da faixa considerada ótima para compostagem de acordo com a literatura. Conclui-se que o processo foi eficiente e que a utilização de cinza é favorável pois a mesma fornece nutrientes aos microrganismos, acelerando a atividade microbiana e gerando, consequentemente, maior aquecimento no interior da leira.

Palavras-chave: Temperatura, Lodo de Laticínio, Cinza de Caldeira. 


\section{INTRODUÇÃO/OBJETIVO}

A produção agroindustrial no Brasil é de grande porte. De acordo com dados do Instituto Brasileiro de Geografia e Estatística (IBGE, 2015), são produzidos no Brasil cerca de 35 bilhões de litros de leite anualmente. Uma indústria de laticínio gera de 0,5 a 2,0 litros de efluente por litro de leite e produz em média $300.000 \mathrm{~L}$ de soro por dia, o que polui o equivalente a uma cidade com 150.000 habitantes (SILVA, 2011). O resíduo oriundo da indústria de laticínio é rico em matéria orgânica e quando descartado de maneira incorreta pode degradar o meio ambiente. Uma maneira de reciclar este resíduo de maneira ambientalmente e economicamente viável é a compostagem.

A compostagem é uma técnica de tratamento de resíduos sólidos que promove a estabilização da matéria orgânica e resulta na redução significativa do volume e peso dos resíduos. Dentre as vantagens deste processo, o Ministério do Meio Ambiente (MMA, 2010) destaca a produção de um composto rico em nutrientes e mais assimilável às plantas, portanto, de interesse agronômico.

Para que o processo de compostagem aconteça deve-se promover a mistura de pelo menos dois resíduos: um que seja a fonte de carbono e outro a fonte de nitrogênio, em proporções que variam de 30 a 40 partes de carbono para uma parte de nitrogênio. O sucesso do processo depende de fatores como umidade, aeração, tamanho de partículas, pH, microrganismos (KIHEL, 1985; PEREIRA NETO, 1996). Deste modo, ao longo do período de compostagem é importante que sejam monitorados diversos parâmetros físico-químicos, dentre eles, a temperatura.

O objetivo deste trabalho é comparar o comportamento da temperatura de duas leiras de compostagem contendo lodo de laticínios, capim, borra de café, na ausência e presença de cinza de caldeira, a partir de alta frequência de coleta de dados.

\section{METODOLOGIA}

O experimento foi conduzido na Universidade Tecnológica Federal do Paraná, Campus Londrina, Brasil, $23^{\circ} 18^{\prime} 32,1^{\prime \prime} \mathrm{S}$ de latitude, $51^{\circ} 07^{\prime} 00,1^{\prime}$ ' W de longitude e altitude 

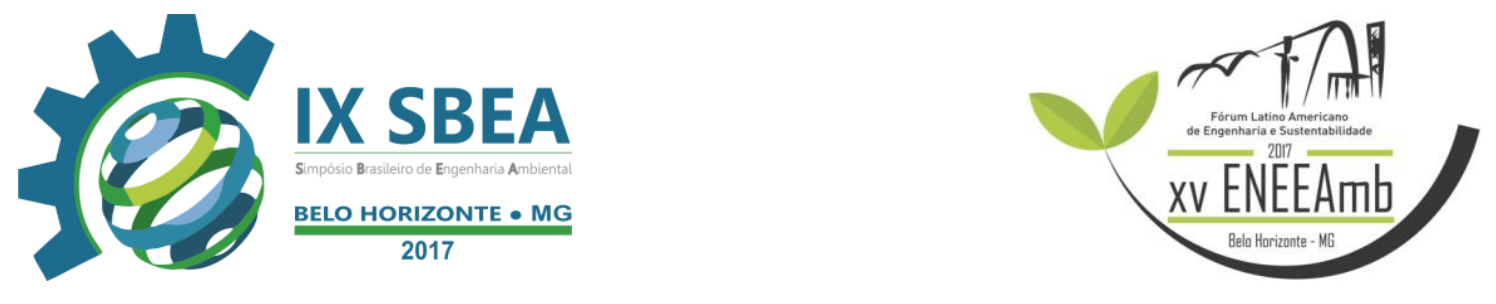

média de 610 metros. A compostagem ocorreu em ambiente protegido da chuva na casa de vegetação sobre piso concretado. Foram montadas duas leiras - L1 e L2 - contendo, em cada, lodo de laticínios (30L), capim (75L) e borra de café (45L), conforme a estratificação indicada na Figura 1. Em uma delas (L2), foi adicionada cinza de caldeira (15L).

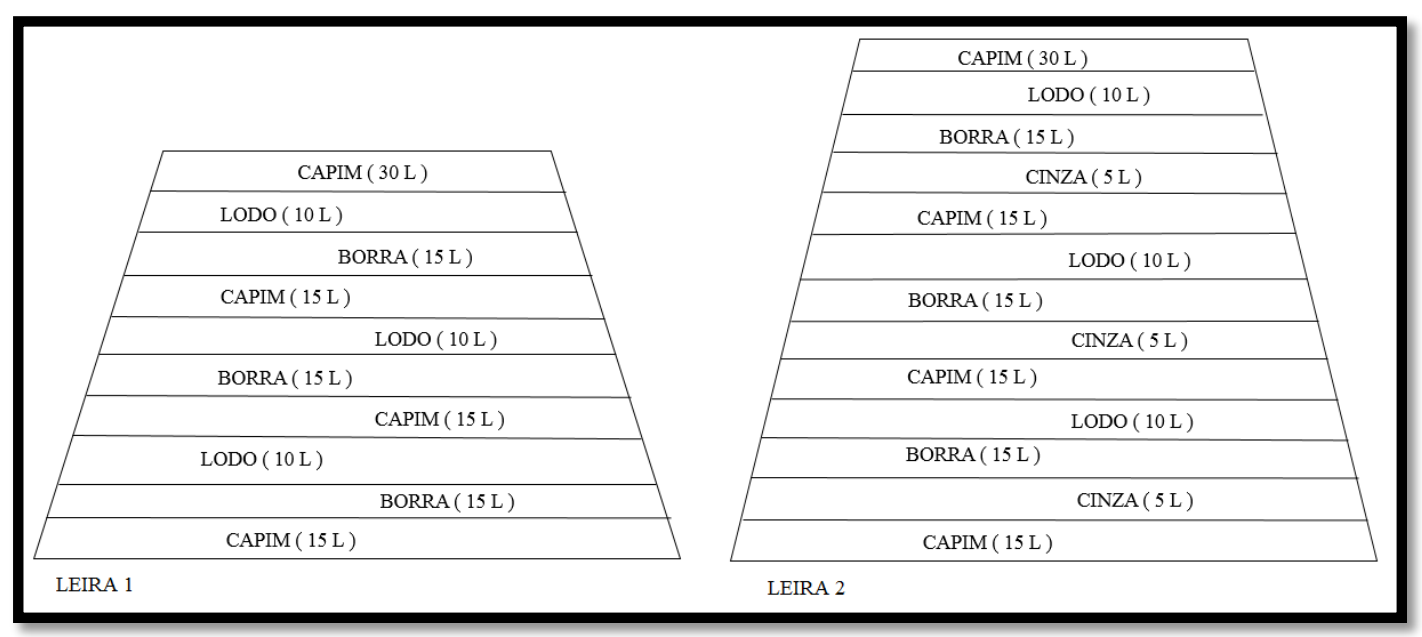

Figura 1 - Composição das leiras de compostagem.

Fonte: Próprios autores.

As leiras foram mantidas nesta estratificação por 5 dias, sendo que no sexto ocorreu o revolvimento, realizado de forma manual, com auxílio de pás e enxadas.

A temperatura foi monitorada por meio de um sistema de coleta automatizada de dados. Foram inseridos, em cada reator, três sensores em pontos distintos, e a coleta de temperatura ocorreu a cada $15 \mathrm{~min}$. Todos os sensores foram ligados a uma placa de arduíno (Figura 2), equipada de sistema registrador de dados (datalogger) junto de um sistema de tempo real que fornece a hora, o minuto e o segundo da aquisição de dados. 

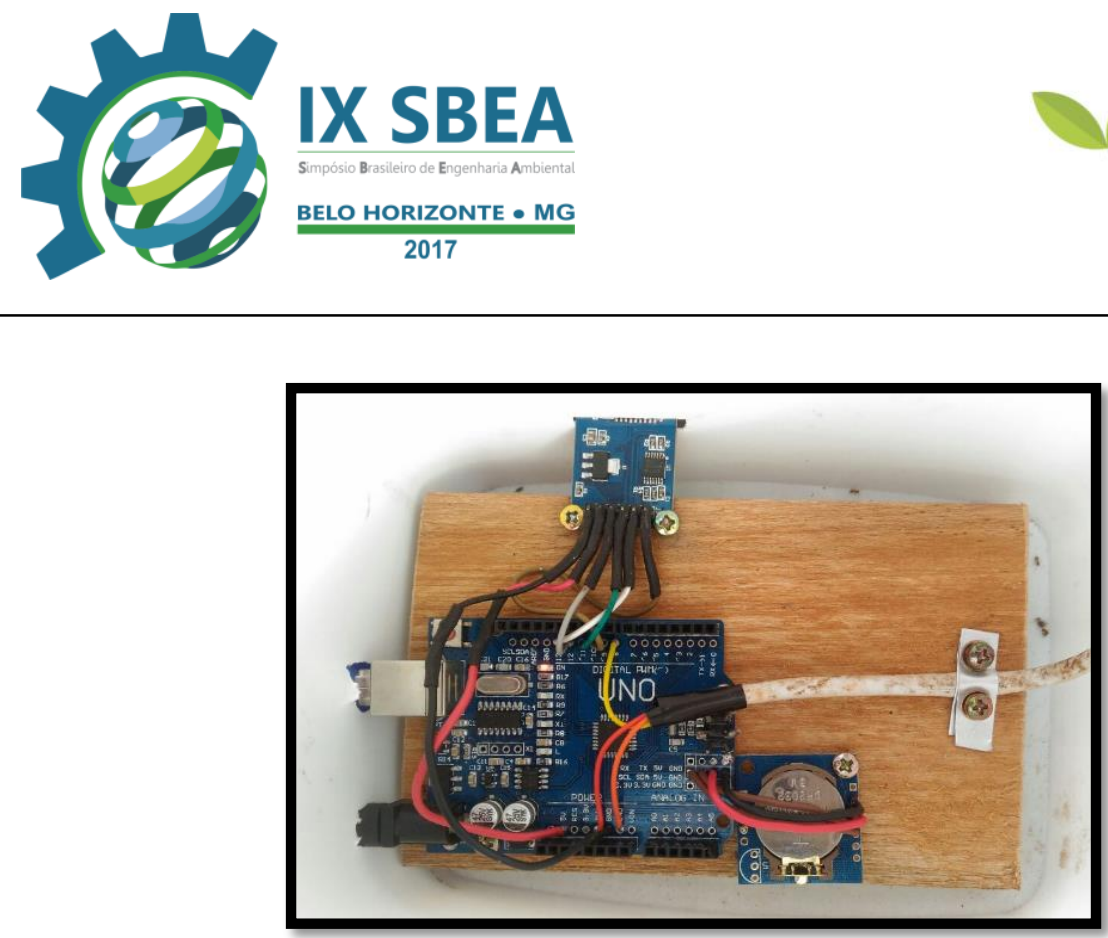

Figura 2 - Arduíno.

Fonte: Próprios autores.

$\mathrm{O}$ pH foi monitorado após 10 dias de compostagem, a partir de uma amostra de $10 \mathrm{~g}$ de composto, dissolvida em $100 \mathrm{~mL}$ de água destilada e agitada no shaker por $30 \mathrm{~min}$. Em seguida, manteve-se a solução em repouso por 1 hora e foi medido o $\mathrm{pH}$ do sobrenadante com auxilio de um peagâmetro (TEDESCO, 1995).

Realizou-se análise de variância, ao nível de 5\% de significância, com os dados médios das temperaturas diárias e as médias foram comparadas entre si pelo teste de Tukey.

\section{RESULTADOS E DISCUSSÃO}

Na Tabela 1, pode-se observar a análise de variância das temperaturas no decorrer dos 10 primeiros dias de compostagem.

Tabela 1 - Análise de variância das temperaturas durante os primeiros 10 dias de compostagem.

\begin{tabular}{cccccc}
\hline Causa de variação & Grau de liberdade & Soma de quadrado & Quadrado médio & $\mathrm{F}_{0}$ & $p$-valor \\
\hline Leira & 1 & 12051 & 12051,000 & 6296,362 & 0,000 \\
Dia & 9 & 90220 & 10024,444 & 5237,535 & 0,000 \\
Interação & 9 & 1516 & 168,444 & 88,008 & $<0,001$ \\
Resíduo & 2650 & 5072 & 1,914 & & \\
\hline Total & 2669 & 108859 & & \\
\hline \multicolumn{7}{c}{}
\end{tabular}

Fonte: Próprios autores. 


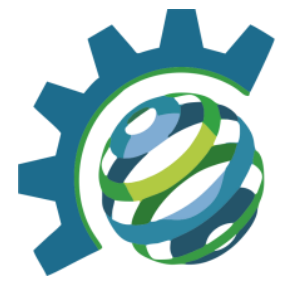

Na Tabela 1 é possível observar que ao nível de 5\% de significância, existe efeito de interação da temperatura entre os dias e a leira. Portanto, o intuito na Tabela 2 é de apresentar o efeito das leiras dentro dos níveis de temperatura de cada dia.

Tabela 2 - Efeito das leiras dentro dos níveis de temperatura de cada dia.

\begin{tabular}{ccccc}
\hline Dia & Leira & TM & ER & Grupo \\
\hline 1 & L2 & 29,049 & 0,113 & A \\
1 & L1 & 28,583 & 0,072 & B \\
\hline 2 & L2 & 38,056 & 0,242 & A \\
2 & L1 & 33,272 & 0,139 & B \\
\hline 3 & L2 & 44,421 & 0,078 & A \\
3 & L1 & 37,683 & 0,099 & B \\
\hline 4 & L2 & 47,701 & 0,083 & A \\
4 & L1 & 43,405 & 0,183 & A \\
\hline 5 & L2 & 48,734 & 0,056 & A \\
5 & L1 & 45,416 & 0,027 & B \\
\hline 6 & L2 & 47,53 & 0,154 & A \\
6 & L1 & 44,333 & 0,136 & B \\
\hline 7 & L2 & 52,539 & 0,076 & B \\
\hline 7 & L1 & 48,179 & 0,034 & A \\
\hline 8 & L2 & 49,825 & 0,076 & B \\
\hline 8 & L1 & 46,244 & 0,114 & A \\
\hline 9 & L2 & 45,91 & 0,099 & B \\
\hline 9 & L1 & 40,212 & 0,122 & A \\
\hline 10 & L2 & 41,385 & 0,08 & B \\
\hline 10 & L1 & 36,276 & 0,123 & \\
\hline
\end{tabular}

Nota 1: TM - Temperatura Média; ER - Erro Padrão.

Nota 2: Letras diferentes na mesma coluna indicam diferença estatística, sendo comparado cada tratamento, pelo teste de Tukey ao nível de 5\% de significância.

Fonte: Próprios autores.

De acordo com a Tabela 2, pode-se observar que há diferença estatística, a 5\% de significância, dos valores das temperaturas médias entre as leiras, nos níveis dos dias, exceto para o quarto dia de compostagem.

A partir da Figura 3, é possível visualizar a variação de temperatura média no decorrer dos 10 primeiros dias de compostagem, e avaliar a temperatura ambiente, para análise comparativa. 

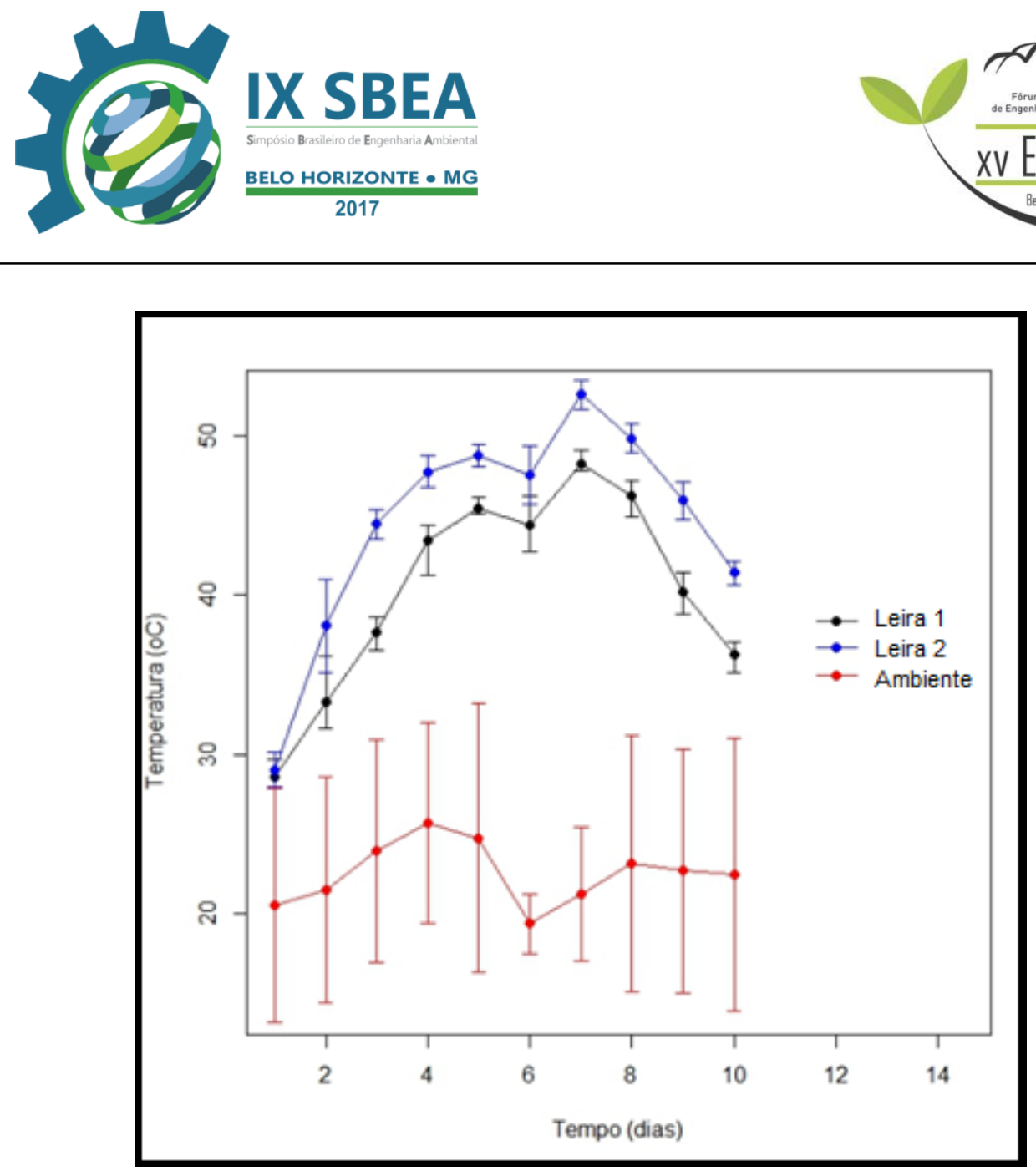

Figura 3: Variação da temperatura ao longo dos 10 primeiros dias de compostagem. Fonte: Próprios autores.

Analisando a estatística da Tabela 2 e seus respectivos valores traçados na Figura 3, é possível observar as fases da compostagem apresentadas por Fernandes (1999) que afirma que a fase mesofílica ocorre normalmente entre 15 a $45^{\circ} \mathrm{C}$ e a fase termofílica ocorre de 45 e $65^{\circ} \mathrm{C}$. Até o terceiro dia, nota-se, portanto, a fase mesofílica e, em seguida a termofílica em ambos os tratamentos. A fase termofílica é de suma importância, pois é nesta fase que ocorre a maior atividade microbiana e os microrganismos patogênicos podem ser eliminados (PEREIRA NETO \& STENTIFORD, 1992). Observa-se também que em todos os dias a temperatura das leiras esteve acima da ambiente, apontando a atividade microbiana decorrente do processo. Kiehl (1985) explica que vários fatores podem influenciar na temperatura de uma leira, como umidade, aeração e relação C/N. Avaliando o primeiro dia de revolvimento, que aconteceu no sexto dia de compostagem, é visível que no dia posterior houve um pico de temperatura atingindo certa de $54^{\circ} \mathrm{C}$ (L2). Barreira (2005) e Inácio \& Miller (2009) explicam que tal comportamento acontece, pois o revolvimento fornece oxigênio ao meio, tornando o ambiente aeróbio, o que propicia plena atividade microbiana. Na comparação entre tratamentos verificou- 

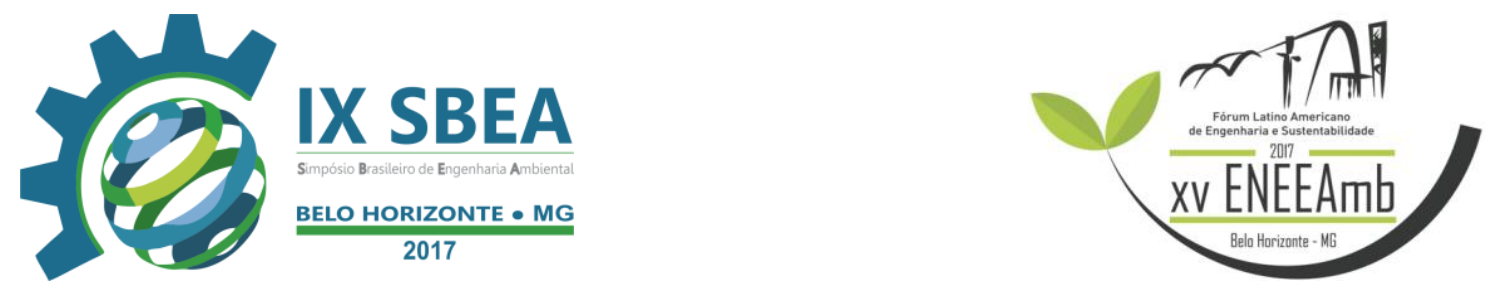

se que a cinza de caldeira favorece na atividade microbiana (L2), o que corrobora com as características vistas por Silva (2011), que afirma em seu estudo que a cinza fornece macro e micronutrientes de fundamental importância para o desenvolvimento de um composto rico.

O pH encontrado no resíduo inicial foi 7,79 e 8,87 para L1 e L2, respectivamente. Segundo Pereira Neto (2007), a faixa de pH considerada ótima para o desenvolvimento dos microrganismos responsáveis pela compostagem situa-se entre 4,5 e 9,5, uma vez que a maioria das enzimas se encontram ativas nesta faixa de $\mathrm{pH}$.

\section{CONCLUSÕES/RECOMENDAÇÕES}

Conclui-se que leiras com a presença de cinza auxiliam na atividade microbiana, o que interfere diretamente no aquecimento da leira, fornecendo subsídios na composição de um composto mais rico nutricionalmente. As leiras atingiram fase termofílica logo nos primeiros dias de compostagem e a aeração é de fundamental importância para uma melhor atividade microbiana.

\section{REFERÊNCIAS BIBLIOGRÁFICAS}

BARREIRA, L. P.; Avaliação das usinas de compostagem do estado de São Paulo em função da qualidade dos compostos e processos de produção. 2005. 204f. Tese (Doutorado em Saúde Ambiental) - Universidade São Paulo, São Paulo, 2005.

BRASIL. MINISTÉRIO DO MEIO AMBIENTE (MMA). Gestão de resíduos orgânicos. (2010). Disponível em: http://www.mma.gov.br/cidadessustentaveis/residuossolidos/gest\%C3\%A3o-de-res\%C3\%ADduos-org\%C3\%A2nicos.

Acesso em 25 maio 2017.

FERNANDES, P. A. L. Estudo Comparativo e Avaliação de Diferentes Sistemas de Compostagem de Resíduos Sólidos Urbanos. Coimbra, 128 p., 1999. Dissertação (Mestrado) - Universidade de Coimbra Faculdade de Ciências e Tecnologia Departamento de Engenharia Civil.

Instituto Brasileiro de Geografia e Estatística (IBGE), 2015, Disponível em: http://biblioteca.ibge.gov.br/visualizacao/periodicos/84/ppm_2015_v43_br.pdf. Acesso em: 29 maio 2017. 


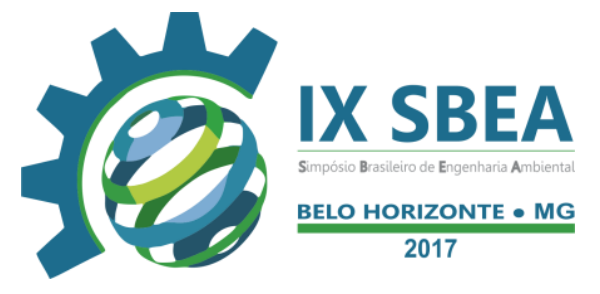

INÁCIO, C. T.; MILLER, P. R. M.; Compostagem: ciência e prática para gestão de resíduos orgânicos. Rio de Janeiro: Embrapa Solos, 2009, 156p.

KIEHL, E. J. Fertilizantes orgânicos. Piracicaba, Brasil, Editora Agronômica Ceres, 1985. $492 \mathrm{p}$.

PEREIRA NETO, João Tinoco Manual de compostagem: processo de baixo custo. Universidade de Viçosa. Viçosa. 2007.

PEREIRA NETO, J.T. Manual de compostagem processo de baixo custo. Belo Horizonte: UNICEF. 56p. 1996.

PEREIRA NETO, J.T.; STENTIFORD, E.I. Aspectos epidemiológicos da compostagem. Revista de Biologia, Uberlândia, v.1, n.1, p.1-6, 1992.

SILVA, D. J. DA. Resíduos na indústria de laticínios. Série de Gestão Ambiental - ViçosaMG (2011). Disponível em: https://www2.cead.ufv.br/sgal/files/apoio/saibaMais/saibaMais2.pdf. Acesso: 29 maio 2017.

TEDESCO, M. J.; GIANELLO, C.; BISSANI, C. A.; BOHNEN, H.; VOLKWEISS, S. J. Análises de solo, plantas e outros materiais. 2. Ed. Porto Alegre: Dpto de solos da UFRGS. 1995, 175p. 